\title{
Ultra high risk status and transition to psychosis in $22 q 11.2$ deletion syndrome
}

Maude Schneider ${ }^{1,2}$, Marco Armando ${ }^{1,3}$, Maria Pontillo ${ }^{3}$, Stefano Vicari ${ }^{3}$, Martin Debbané ${ }^{1,4,5}$, Frauke Schultze-Lutter ${ }^{6}$, Stephan Eliez ${ }^{1,7}$

${ }^{1}$ Office Médico-Pédagogique Research Unit, Department of Psychiatry, School of Medicine, University of Geneva, Geneva, Switzerland; ${ }^{2}$ Center for Contextual Psychiatry, Department of Neuroscience, KU Leuven, Leuven, Belgium; ${ }^{3}$ Child and Adolescence Neuropsychiatry Unit, Department of Neuroscience, Bambino Gesù Children Hospital, Rome, Italy; ${ }^{4}$ Developmental Clinical Psychology Unit, Faculty of Psychology, University of Geneva, Geneva, Switzerland; ${ }^{5}$ Research Department of Clinical, Educational and Health Psychology, University College London, London, UK; ${ }^{6}$ University Hospital of Child and Adolescent Psychiatry and Psychotherapy, University of Bern, Bern, Switzerland; ${ }^{7}$ Department of Genetic Medicine and Development, School of Medicine, University of Geneva, Geneva, Switzerland 
The $22 \mathrm{q} 11.2$ deletion syndrome (22q11DS) is characterized by high rates of psychotic symptoms and schizophrenia, making this condition a promising human model for studying risk factors for psychosis. We explored the predictive value of ultra high risk (UHR) criteria in a sample of patients with 22q11DS. We also examined the additional contribution of socio-demographic, clinical and cognitive variables to predict transition to psychosis within an interval of $32.5 \pm 17.6$ months after initial assessment. Eighty-nine participants with 22q11DS (age range: 8-30 years; mean 16.1 \pm 4.7 ) were assessed using the Structured Interview for Psychosis-Risk Syndromes. Information on Axis I diagnoses, internalizing and externalizing symptoms, level of functioning and IQ was also collected. At baseline, 22 (24.7\%) participants met UHR criteria. Compared to those without a UHR condition, they had a significantly lower functioning, more frequent anxiety disorders, and more severe psychopathology. Transition rate to psychosis was $27.3 \%$ in UHR and $4.5 \%$ in non-UHR participants. Cox regression analyses revealed that UHR status significantly predicted conversion to psychosis. Baseline level of functioning was the only other additional predictor. This is the first study investigating the predictive value of UHR criteria in 22q11DS. It indicates that the clinical path leading to psychosis is broadly comparable to that observed in other clinical high-risk samples. Nevertheless, the relatively high transition rate in non-UHR individuals suggests that other risk markers should be explored in this population. The role of low functioning as a predictor of transition to psychosis should also be investigated more in depth.

Key words: 22q11.2 deletion syndrome, schizophrenia, clinical high risk state, ultra high risk criteria, transition to psychosis, level of functioning 
In the past 20 years, there has been an increasing interest in people presenting with potentially prodromal symptoms of psychosis, i.e. with a clinical high risk state. Criteria have been developed to identify that high risk state: the ultra high risk (UHR) and the basic symptom criteria $^{1,2}$. A recent meta-analysis reported an ability of UHR criteria to detect transition to psychosis within two years in $20 \%$ of individuals in clinical samples ${ }^{3}$. Yet, although persons with a clinical high risk state have a significantly increased risk of developing psychosis, many will not develop a psychotic disorder. The specificity of clinical high risk assessments hence remains relatively low ${ }^{4}$.

Studies of genetic syndromes associated with increased risk of schizophrenia have become increasingly important. Among these genetic conditions, 22q11.2 deletion syndrome (22q11DS) is particularly valuable ${ }^{5}$. This syndrome is characterized in most cases by a microdeletion of 3 million base pairs on chromosome 22 band q11, and has an estimated prevalence of 1:2.000-4.000 live births $^{6}$. From a clinical perspective, 22q11DS is associated with high rates of psychiatric disorders, especially schizophrenia ${ }^{7}$. While 23 to $45 \%$ of adolescents report transient psychotic experiences ${ }^{8-}$ ${ }^{11}$, up to $40 \%$ of affected adults are diagnosed with a psychotic disorder ${ }^{7}$. Moreover, 22q11DS was found in 0.3 to $2.0 \%$ of patients with schizophrenia ${ }^{12-14}$, with rates of up to $5.7 \%$ in patients with childhood-onset schizophrenia ${ }^{15}$. Taken together, these findings indicate that 22q11DS is a highly relevant genetic risk factor for schizophrenia and the most promising human model for studying risk factors and states at risk for schizophrenia ${ }^{5}$.

Several studies have investigated prodromal symptoms in patients with 22q11DS, reporting rates between 45 and $56 \%$ for UHR symptoms and between 10 and $21 \%$ for UHR criteria

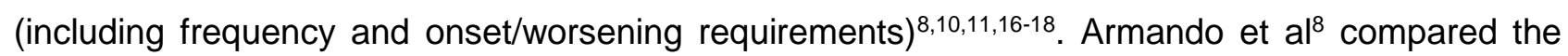
symptom profile of UHR patients with $(\mathrm{N}=30)$ vs. without $(\mathrm{N}=81) 22 q 11 \mathrm{DS}$ and found no significant group difference in positive symptoms, while negative symptoms were more severe in patients with 22q11DS.

Yet, few studies have prospectively investigated risk factors for psychosis in the 22q11DS population. Gothelf et $\mathrm{al}^{19}$ found that anxiety disorder and lower full-scale IQ at baseline, and a greater decline in verbal IQ were the best predictors of transition to psychosis. In line with these findings, Vorstman et $\mathrm{al}^{20}$ reported that an early cognitive decline, particularly in verbal IQ, was a robust predictor of psychosis. Finally, a recent study highlighted the role of poor premorbid adjustment during childhood and adolescence in the development of UHR symptoms and fullblown psychosis ${ }^{21}$. No study, however, has longitudinally examined the predictive value of UHR criteria in people with 22q11DS.

We investigated prospectively, in a large group of patients with 22q11DS over an average period of 32 months, the predictive value of UHR criteria as well as potential other predictors of conversion to psychosis. We hypothesized that the predictive value of UHR criteria would be comparable to that found in other clinical samples, albeit expecting an overall higher transition rate, 
given the higher prevalence of psychotic disorders in 22q11DS. Secondly, we expected that low baseline verbal IQ, the presence of an anxiety disorder, and low baseline level of functioning would increase the predictive accuracy in addition to the presence of an UHR condition.

\section{METHODS}

\section{Participants}

We included 89 participants (56 from Geneva and 33 from Rome) with a genetically confirmed 22q11DS diagnosis, aged between 8 and 30 years (mean 16.1 \pm 4.7 ) at baseline. Having a psychotic disorder at baseline was an exclusion criterion. Children were assessed from the age of 8 onwards, as previous studies reported the presence of psychotic symptoms in young children with 22q11DS 9 . Participants were followed-up over a mean period of $32.5 \pm 17.6$ months (range: $12-$ 85).

Participants from Geneva were recruited through advertisements in patient associations or word of mouth; those from Rome were referred from the Genetic Clinical Unit of the Bambino Gesù Hospital or recruited through advertisement in patient associations. Written informed consent from the participants and their parents was collected at both sites under protocols approved by local institutional ethical review boards.

Compared to the Geneva cohort, participants from Rome were younger (mean age 14.3 \pm 5.1 vs. $17.1 \pm 4.2$ years, $t=2.89, p=0.005)$ and had a higher full-scale $I Q(84.5 \pm 10.9$ vs. $72.2 \pm 10.0$, $t=-5.44, p<0.001$ ), while the gender distribution at baseline was similar (females: $51.5 \%$ vs. $55.4 \%$, $\left.\chi^{2}=0.12, p=0.725\right)$.

\section{Assessments}

All participants completed the Structured Interview for Psychosis-Risk Syndromes ${ }^{22}$ to assess the severity of positive, negative, disorganization and general symptoms, as well as the presence of UHR symptoms (any P1-P5 $\geq 3$ ) and UHR criteria (i.e., attenuated psychotic symptoms (APS), brief limited intermittent symptoms (BLIPS), or genetic risk and functional decline (GRFD) criteria). We also explored the rate of participants meeting criteria for perceptive (P4) and non-perceptive (P1, P2, P3 or P5) APS or BLIPS ${ }^{23}$. For the global assessment of functioning, the Childhood Global Assessment Scale (CGAS) ${ }^{24}$ or the Global Assessment of Functioning (GAF) was used.

The presence of any Axis I DSM-IV psychiatric disorder was assessed using structured clinical interviews. In both cohorts, the Structured Clinical Interview for Axis I DSM-IV (SCID-I) ${ }^{25}$ was administered to adult participants and their parents. In Geneva, parents of participants below 18 
years completed the Diagnostic Interview for Children and Adolescents - Revised (DICA-IV) ${ }^{26}$ and diagnoses were confirmed with the participants. The psychotic disorders supplement of the Schedule for Affective Disorders and Schizophrenia for School-Age Children - Present and Lifetime version (K-SADS-PL) ${ }^{27}$ was also administered. In Rome, the K-SADS-PL, including the psychotic disorders supplement, was used for children and adolescents.

Intellectual functioning was assessed by trained psychologists at both time points using the Wechsler Intelligence Scale for Children - third edition (WISC-III) ${ }^{28}$ or the Wechsler Adult Intelligence Scale - third edition (WAIS-III) ${ }^{29}$. Verbal IQ, performance IQ and full-scale IQ were used as indicators of intellectual functioning.

Parents of all participants completed the Child Behavior Checklist $(\mathrm{CBCL})^{30}$ or the Adult Behavior Checklist $(\mathrm{ABCL})^{31}$. Internalizing, externalizing and total problems T-scores were used as global measures of the severity of psychopathology.

\section{Statistical analyses}

All statistical analyses were performed using SPSS version 21. Cross-sectional group comparisons between participants with and without UHR criteria at baseline were performed using independent t-tests, Mann-Whitney $U$, or $\chi^{2}$ tests. Because of the variable interval between baseline and follow-up, we conducted a Cox regression analysis to determine whether the presence of any UHR state at baseline (UHR status) significantly predicted conversion to psychosis. Furthermore, we used Cox regression analyses to examine whether perceptive and non-perceptive APS/BLIPS were both predictors of conversion to psychosis. In case of two significant models, both predictors were entered in a stepwise Cox regression analysis.

We then examined the contribution of additional variables at baseline to improve the UHRbased prediction model. Potential predictors included: demographic characteristics (age, gender), clinical parameters (presence of any anxiety, affective or behavioral disorder, CGAS/GAF scores, CBCL/ABCL internalizing, externalizing and total problems T-scores, and severity of positive, negative, disorganization and general symptoms), and cognitive variables (verbal, performance and full-scale IQ).

A multiple step approach was adopted in order to derive a parsimonious model. First, each predictor was entered individually in a series of Cox regression analyses and selected for further analyses when the Wald statistic was significant at a liberal level $(p<0.05)$. Next, each selected predictor was entered in a multiple Cox regression analysis, with UHR status always included as a predictor. Variables were further selected if the Wald statistic was significant $(p<0.05)$ for both variables (UHR status and the additional predictor), indicating that the additional predictor contributed to the improvement of prediction without decreasing the predictive value of UHR status. If more than one predictor met the above-mentioned criteria, all predictors were analyzed 
together using a forward and backward Cox regression analysis to exclude effects of blocking. The maximum number of predictors entering the final model was limited to a 1:5 ratio of number of predictors to event. The proportional hazard assumption was tested at each step prior to each Cox regression following the procedure described by Kleinbaum and Klein ${ }^{32}$.

\section{RESULTS}

\section{Baseline characteristics}

Twenty-two (24.7\%) participants met UHR criteria at baseline (UHR+) and 67 (75.3\%) did not (UHR-). Compared to UHR- participants, UHR+ were more frequently under antipsychotic medication and diagnosed with an anxiety disorder, had more severe positive symptoms (all $P$ subscales except grandiosity) and internalizing and externalizing symptoms, and had a lower functioning at baseline, although not a higher rate of functional deficit (CGAS/GAF score <70) (Table 1). The most frequent UHR condition was APS ( $\mathrm{N}=15 ; 68.2 \%)$, followed by GRFD ( $\mathrm{N}=6$; $27.3 \%)$ and BLIPS ( $\mathrm{N}=2 ; 9.1 \%)$; only one participant (4.2\%) met criteria for both APS and GRFD. Among the 17 participants with APS or BLIPS, four (23.5\%) presented with perceptive, six (35.3\%) with non-perceptive, and seven (41.2\%) with both perceptive and non-perceptive APS/BLIPS.

In addition, 10 (11.2\%) participants experienced UHR symptoms - six on item P4 (perceptual abnormalities/hallucinations), two on item P2 (suspiciousness/persecutory ideas), and two on several items - but failed to meet frequency $(\mathrm{N}=6)$ or both frequency and onset/worsening requirements $(\mathrm{N}=4)$. Altogether, the prevalence of UHR symptoms (regardless of frequency and onset/worsening requirements) was $36.0 \%$.

\section{Outcome}

Altogether, nine (10.1\%) participants had converted to psychosis at follow-up, four being minors (<18 years) and five adults at baseline (Table 2). The six UHR+ converters included three of those diagnosed with APS (out of $15,20.0 \%$ ), both of those diagnosed with BLIPS (100\%), and one of those diagnosed with GRFD (out of six, 16.7\%). Of the three false-negative cases (i.e., UHR- participants at baseline who converted to psychosis), one had reported UHR symptoms at baseline which did not meet frequency and worsening/onset criteria. Five participants (all UHR+) were receiving antipsychotic medication at baseline; three of them converted to psychosis at follow-up. 
None of the ten participants who remitted from UHR status (i.e., UHR+ at baseline, but UHRat follow-up) had received antipsychotic medication at either baseline or follow-up. Furthermore, four (4.5\%) participants had a new onset of UHR criteria at follow-up.

\section{Predictors of conversion to psychosis}

UHR status at baseline was a significant predictor of transition to psychosis $(\beta=1.823$, $\mathrm{SE}=0.733$, Wald $(\mathrm{df}=1)=6.181, \mathrm{p}=0.013 ; \operatorname{Exp}(\beta)=6.188,95 \% \mathrm{Cl}: 1.471-26.033)$. Furthermore, presence of both perceptive APS/BLIPS $(\beta=1.644, S E=0.737$, Wald $(d f=1)=4.975, p=0.026$; $\operatorname{Exp}(\beta)=5.178,95 \% \mathrm{Cl}: 1.221-21.961)$ and non-perceptive APS/BLIPS ( $\beta=3.397, \mathrm{SE}=0.876$, Wald $(d f=1)=15.021, p<0.001 ; \operatorname{Exp}(\beta)=29.868,95 \% \mathrm{Cl}: 5.360-166.432)$ significantly predicted transition to psychosis. When both variables were entered in a stepwise Cox regression analysis, only the presence of non-perceptive APS/BLIPS remained in the final model.

With regard to additional predictors, CGAS/GAF at baseline remained the only significant predictor after the two selection steps. The final model (Table 3), including UHR status and CGAS/GAF as predictors, was highly significant $\left(-2 L L=48.768, \chi^{2}(d f=2)=15.329, p<0.001\right)$. Cumulative hazard rates of the model were 0.015 at two years, 0.024 at three years and 0.113 at four years.

\section{DISCUSSION}

\section{UHR symptoms and criteria}

Altogether, 32 (36.0\%) participants reported at least one UHR symptom (APS or BLIPS) regardless of the frequency and onset/worsening requirements. Twenty-two of them (24.7\%) fully met UHR criteria (i.e., including frequency and onset/worsening requirements). Both rates are broadly consistent with previous studies in 22q11DS, reporting rates between 45 and $56 \%$ for UHR symptoms and between 10 and $21 \%$ for UHR criteria ${ }^{10,11,16-18,33}$. Thus, our findings confirm that patients with 22q11DS are at increased risk of experiencing attenuated symptoms of psychosis, regardless of transition to psychosis ${ }^{23,34}$. Indeed, recent estimates from the general population were between 7.3 and $9.9 \%$ for lifetime UHR symptoms and between 0.4 and $1.3 \%$ for current UHR criteria.

In the present sample, APS was the most prevalent UHR condition (68.2\%), followed by GRD $(27.3 \%)$ and BLIPS (9.1\%). While the preponderance of APS and the low frequency of BLIPS is consistent with findings from other clinical high risk populations ${ }^{3,35-37}$, GRFD was more frequent than in most clinical UHR samples ${ }^{3}$. 
In line with earlier findings ${ }^{9}$, patients meeting UHR criteria exhibited lower level of functioning and higher levels of internalizing and externalizing symptoms, and were more frequently diagnosed with an anxiety disorder. These findings highlight that the presence of a UHR status in 22q11DS, similarly to other clinical populations ${ }^{3}$, is in itself a condition that aggravates the clinical picture and, consequently, requires clinical attention, irrespective of any potential future transition to psychosis.

Although a European guidance on early intervention in clinical high risk states does not recommend the use of antipsychotics as first line treatment in patients with $\mathrm{UHR}^{38}$, nearly a quarter of UHR+ but none of UHR- participants were receiving antipsychotic medication at baseline. This practice might have been linked to the treating clinicians' awareness of UHR symptoms and increased risk of psychosis in this population, and could be interpreted as a psychosis-prevention approach. However, antipsychotics might have been also prescribed for other behavioral problems, such as severe anxiety or externalizing symptoms, in this more symptomatic group.

\section{Outcome}

We observed a transition rate to psychosis of $27.3 \%$ among UHR+ participants, which is comparable to previous reports in other clinical samples ${ }^{3,39}$. Furthermore, with only $4.5 \%$ of UHRparticipants developing psychosis, we found that the UHR status significantly predicted transition to psychosis in this specific subgroup of patients. However, in light of the increased risk of psychosis recognized in this population, it might be surprising that the transition rates were "only" in line with those of other clinical samples and not considerably higher ${ }^{40}$.

Several reasons may explain this finding. First, the mean age of UHR+ participants in our sample was 16 years, with $23.6 \%$ being 12 years or younger and $53.9 \%$ being 15 years or younger. In children and younger adolescents, the clinical significance as well as the psychosispredictive value of UHR criteria, especially of APS, was reported to be significantly lower than in individuals aged 16 or older ${ }^{3-5,41}$. Thus, the rather high proportion of participants below 16 years might have lowered the overall transition rate considerably. Second, the remission rate from UHR status in non-converters (62.5\%) was in the upper range of what is typically described ${ }^{42}$, suggesting that UHR symptom fluctuation is very common in this population ${ }^{19}$. Several characteristics of the 22q11DS, such as intellectual disability and heightened anxiety levels, and their impact on adaptive functioning and everyday living skills, might indicate that stress sensitivity significantly influences variability in symptom severity ${ }^{43,44}$. Importantly, the interplay of these factors in daily life will have to be examined in future studies as they carry potentially crucial clinical implications.

Although the number of participants meeting criteria for each specific UHR condition (APS, BLIPS and GRFD) remains limited, we observed that a higher percentage of participants with BLIPS (100\%) converted to psychosis, followed by APS (20.0\%), and GRFD (16.7\%). This pattern 
falls in line with results from a recent meta-analysis reporting the highest transition risk for BLIPS, and the lowest for GRFD ${ }^{3}$. Yet, while our numbers were consistent with the pooled transition rate for APS reported in that meta-analysis $(17.4 \%$ at two and $29.1 \%$ at three years), the reported pooled transition rates for BLIPS (46.6\% at two and 51.8\% at three years) and GRFD (1.9\% at two and $1.4 \%$ at three years) were lower in that meta-analysis than those found in the present sample ${ }^{3}$.

We found that non-perceptive APS/BLIPS were a stronger predictor of transition to psychosis than perceptive APS/BLIPS. This finding is in line with previous reports of a low clinical significance of perceptive APS in children and adolescents from the general population ${ }^{23,45,46}$. This highlights the need for further studies examining UHR criteria and symptoms in relation to age in patients with 22q11DS.

The rate of false negative cases (i.e., UHR- participants who converted to psychosis) was higher $(4.5 \%)$ than that reported in recent meta-analyses $(0.9-1.6 \%)$ on patients seeking help at specialized early psychosis detection services ${ }^{3,4}$. This corroborates the fact that 22q11DS is a psychosis-risk condition in itself and linked to a higher baseline probability to develop psychosis. It also highlights the need of investigating other potential risk markers (e.g., clinical or cognitive) in order to improve the detection of patients who will convert to psychosis.

\section{Other predictors of transition to psychosis}

In addition to UHR criteria, only lower baseline level of functioning, but not the presence of a functional deficit, significantly increased the predictive value of the model in our sample. This finding is in line with several studies that also identified lower functioning scores as a relevant predictor of the onset of psychosis in $\mathrm{UHR}^{41,47-49}$ as well as $22 q 11 \mathrm{DS}$ samples ${ }^{21,50}$. Yet, contrary to other findings in 22q11DS ${ }^{19,20}$, baseline verbal $I Q$ and presence of an anxiety disorder did not increase the predictive value of the model. However, it should be noted that these previous studies never included UHR status as a baseline predictor. Hence, it is possible that including UHR status as a predictor reduced the variance explained by these other factors. Another potential explanation of this finding is that anxiety and verbal IQ decline precede or co-occur with the onset of UHR symptoms but do not predict transition to psychosis. Future studies examining the temporal dynamics of these different risk factors may help testing these hypotheses.

\section{Strengths and limitations}

This is the first study examining the predictive value of UHR criteria in patients with 22q11DS, which constitutes an important first step towards prevention of psychosis in this population. However, the relatively small sample size prevented a more detailed analysis of clinical outcomes 
or of interactions between variables. This limitation should be considered in light of the low prevalence of the syndrome, and is generally found in all longitudinal studies on 22q11DS ${ }^{19}$.

A second limitation is the variable interval between the two assessments, which has been taken into account by the use of Cox regression analyses. This also deals with the fact that the true survival time is unknown in such studies (i.e., some participants are still likely to develop psychosis after the second assessment) ${ }^{32}$.

A third limitation is the variance in age of the participants, that might have influenced some of the results, although it has been reported that the mean age of onset of psychosis in 22q11DS is lowered $^{7,19}$. Furthermore, neither our assessment nor our sample size allowed a detailed analysis of treatment effects. However, this neglect of treatment effects beyond the prescription of antipsychotics is rather the rule than the exception in naturalistic UHR follow-up studies.

\section{CONCLUSIONS}

Our findings suggest that the psychopathological path leading to transition to psychosis in 22q11DS is broadly comparable to that observed in other clinical high risk samples, and confirm that 22q11DS can serve as a good human model for studying risk factors for psychosis.

The relatively high percentage of false negative highlights that our efforts should now focus on investigating other possible, more subtle, risk markers - such as cognitive deficits and basic symptoms - to increase the sensitivity of our predictive model ${ }^{2}$. The role of low functioning as a predictor of transition to psychosis should also be investigated more in depth by distinguishing different areas of functioning.

\section{ACKNOWLEDGEMENTS}

This work was supported by research grants from the Swiss National Science Foundation (grant nos. 324730_121996, 324730_144260) and The National Centre of Competence in Research "Synapsy - The Synaptic Bases of Mental Diseases". Grants from the Brain and Behavior Research Foundation (formerly NARSAD) (grant no. 21278) and the Swiss National Science Foundation (grant no. 162006) also supported the present work. The authors would like to thank all the families who contributed to the study as well as the family associations (Aidel 22, Génération 22, Connect 22, Relais 22, and Creaf 22) for their ongoing support. Special thanks go to S. Menghetti from the Office Médico-Pédagogique Research Unit for coordinating the project in Geneva and C. Di Gilio for leading the Genetic Unit in Rome. M. Schneider and M. Armando contributed equally to this work; F. Schultze-Lutter and S. Eliez share last authorship. 


\section{REFERENCES}

1. Fusar-Poli $P$, Borgwardt $S$, Bechdolf $A$ et al. The psychosis high-risk state: a comprehensive state-ofthe-art review. JAMA Psychiatry 2013;70:107-20.

2. Schultze-Lutter F, Debbané M, Theodoridou A et al. Revisiting the basic symptom concept: toward translating risk symptoms for psychosis into neurobiological targets. Front Psychiatry 2016;7:9.

3. Schultze-Lutter F, Michel C, Schmidt SJ et al. EPA guidance on the early detection of clinical high risk states of psychoses. Eur Psychiatry 2015;30:405-16.

4. Fusar-Poli $P$, Cappucciati $M$, Rutigliano $G$ et al. At risk or not at risk? A meta-analysis of the prognostic accuracy of psychometric interviews for psychosis prediction. World Psychiatry 2015;14:322-32.

5. Insel TR. Rethinking schizophrenia. Nature 2010;468:187-93.

6. Bassett AS, McDonald-McGinn DM, Devriendt $K$ et al. Practical guidelines for managing patients with 22q11.2 deletion syndrome. J Pediatr 2011;159:332-9.

7. Schneider $M$, Debbané $M$, Bassett $A S$ et al. Psychiatric disorders from childhood to adulthood in 22q11.2 deletion syndrome: results from the International Consortium on Brain and Behavior in 22q11.2 Deletion Syndrome. Am J Psychiatry 2014;171:627-39.

8. Armando M, Girardi P, Vicari S et al. Adolescents at ultra-high risk for psychosis with and without $22 q 11$ deletion syndrome: a comparison of prodromal psychotic symptoms and general functioning. Schizophr Res 2012;139:151-6.

9. Debbané M, Glaser B, David MK et al. Psychotic symptoms in children and adolescents with $22 q 11.2$ deletion syndrome: neuropsychological and behavioral implications. Schizophr Res 2006;84:187-93.

10. Schneider M, Van der Linden M, Glaser B et al. Preliminary structure and predictive value of attenuated negative symptoms in 22q11.2 deletion syndrome. Psychiatry Res 2012;196:277-84.

11. Stoddard J, Niendam T, Hendren R et al. Attenuated positive symptoms of psychosis in adolescents with chromosome 22q11.2 deletion syndrome. Schizophr Res 2010;118:118-21.

12. Arinami T. Analyses of the associations between the genes of $22 q 11$ deletion syndrome and schizophrenia. J Hum Genet 2006;51:1037-45.

13. Shprintzen RJ, Karayiorgou M, Morris MA et al. Schizophrenia susceptibility associated with interstitial deletions of chromosome 22q11. Proc Natl Acad Sci USA 1995;92:7612-6.

14. Stefansson $\mathrm{H}$, Rujescu $D$, Cichon $S$ et al. Large recurrent microdeletions associated with schizophrenia. Nature 2008;455:232-6.

15. Sporn A, Addington A, Reiss AL et al. 22q11 deletion syndrome in childhood onset schizophrenia: an update. Mol Psychiatry 2004;9:225-6.

16. Rockers K, Ousley O, Sutton $\mathrm{T}$ et al. Performance on the Modified Card Sorting Test and its relation to psychopathology in adolescents and young adults with 22q11.2 deletion syndrome. J Intellect Disabil Res 2009;53:665-76.

17. Shapiro DI, Cubells JF, Ousley OY et al. Prodromal symptoms in adolescents with $22 q 11.2$ deletion syndrome and schizotypal personality disorder. Schizophr Res 2011;129:20-8.

18. Tang SX, Yi JJ, Calkins ME et al. Psychiatric disorders in 22q11.2 deletion syndrome are prevalent but undertreated. Psychol Med 2013;44:1-11. 
19. Gothelf D, Schneider M, Green T et al. Risk factors and the evolution of psychosis in $22 q 11.2$ deletion syndrome: a longitudinal 2-site study. J Am Acad Child Adolesc Psychiatry 2013;52:1192-203.

20. Vorstman JAS, Breetvelt EJ, Duijff $S N$ et al. Cognitive decline preceding the onset of psychosis in patients with 22q11.2 deletion syndrome. JAMA Psychiatry 2015;72:377-85.

21. Radoeva PD, Fremont $\mathrm{W}$, Antshel KM et al. Longitudinal study of premorbid adjustment in $22 q 11.2$ deletion (velocardiofacial) syndrome and association with psychosis. Dev Psychopathol (in press).

22. McGlashan T, Walsh BC, Woods SW. The psychosis-risk syndrome: handbook for diagnosis and followup. New York: Oxford University Press, 2010.

23. Schimmelmann BG, Michel C, Martz-Irngartinger A et al. Age matters in the prevalence and clinical significance of ultra-high-risk for psychosis symptoms and criteria in the general population: findings from the BEAR and BEARS-kid studies. World Psychiatry 2015;14:189-97.

24. Shaffer D, Gould MS, Brasic J et al. A children's global assessment scale (CGAS). Arch Gen Psychiatry 1983;40:1228-31.

25. Spitzer RL, Williams JB, Gibbon $M$ et al. The Structured Clinical Interview for DSM-III-R (SCID). I: history, rationale, and description. Arch Gen Psychiatry 1992;49:624-9.

26. Reich W. Diagnostic interview for children and adolescents (DICA). J Am Acad Child Adolesc Psychiatry 2000;39:59-66.

27. Kaufman J, Birmaher B, Brent D et al. Schedule for Affective Disorders and Schizophrenia for SchoolAge Children-Present Lifetime Version (K-SADS-PL): initial reliability and validity data. J Am Acad Child Adolesc Psychiatry 1997;36:980-8.

28. Wechsler D. The Wechsler Intelligence Scale for Children - third edition: administration and scoring manual. San Antonio: Psychological Corporation, 1991.

29. Wechsler D. Wechsler Adult Intelligence Scale - III: administration and scoring manual. San Antonio: Psychological Corporation, 1997.

30. Achenbach TM, Rescorla LA. Manual for the ASEBA school-age forms and profiles. Burlington: University of Vermont, Research Center for Children, Youth, and Families, 2001.

31. Achenbach TM, Rescorla LA. Manual for the ASEBA adult forms and profiles. Burlington: University of Vermont, Research Center for Children, Youth and Families, 2003.

32. Kleinbaum DG, Klein M. Survival analysis: a self-learning text. New York: Springer, 2005.

33. Schneider M, Schaer M, Mutlu AK et al. Clinical and cognitive risk factors for psychotic symptoms in 22q11.2 deletion syndrome: a transversal and longitudinal approach. Eur Child Adolesc Psychiatry 2013;23:425-36.

34. Schultze-Lutter F, Michel C, Ruhrmann $S$ et al. Prevalence and clinical significance of DSM-5attenuated psychosis syndrome in adolescents and young adults in the general population: the Bern Epidemiological At-Risk (BEAR) study. Schizophr Bull 2014;40:1499-508.

35. Hartmann JA, Yuen HP, McGorry PD et al. Declining transition rates to psychotic disorder in "ultra-high risk" clients: investigation of a dilution effect. Schizophr Res 2016;170:130-6.

36. Nelson B, Yuen HP, Wood SJ et al. Long-term follow-up of a group at ultra high risk ("prodromal") for psychosis: the PACE 400 study. JAMA Psychiatry 2013;70:793-802.

37. Nelson B, Yuen K, Yung AR. Ultra high risk (UHR) for psychosis criteria: are there different levels of risk for transition to psychosis? Schizophr Res 2011;125:62-8. 
38. Schmidt SJ, Schultze-Lutter F, Schimmelmann BG et al. EPA guidance on the early intervention in clinical high risk states of psychoses. Eur Psychiatry 2015;30:388-404.

39. Fusar-Poli P, Bonoldi I, Yung AR et al. Predicting psychosis: meta-analysis of transition outcomes in individuals at high clinical risk. Arch Gen Psychiatry 2012;69:220-9.

40. Fusar-Poli P, Schultze-Lutter F. Predicting the onset of psychosis in patients at clinical high risk: practical guide to probabilistic prognostic reasoning. Evid Based Ment Health 2016;19:10-5.

41. Cornblatt BA, Carrión RE, Auther A et al. Psychosis prevention: a modified clinical high risk perspective from the Recognition and Prevention (RAP) program. Am J Psychiatry 2015;172:986-94.

42. Simon $A E$, Velthorst $E$, Nieman $D H$ et al. Ultra high-risk state for psychosis and non-transition: a systematic review. Schizophr Res 2011;132:8-17.

43. Angkustsiri $\mathrm{K}$, Leckliter I, Tartaglia $\mathrm{N}$ et al. An examination of the relationship of anxiety and intelligence to adaptive functioning in children with chromosome 22q11.2 deletion syndrome. J Dev Behav Pediatr 2012;33:713-20.

44. Beaton EA, Simon TJ. How might stress contribute to increased risk for schizophrenia in children with chromosome 22q11.2 deletion syndrome? J Neurodev Disord 2011;3:68-75.

45. Bartels-Velthuis AA, Jenner JA, van de Willige $G$ et al. Prevalence and correlates of auditory vocal hallucinations in middle childhood. Br J Psychiatry 2010;196:41-6.

46. Bartels-Velthuis AA, van de Willige $G$, Jenner JA et al. Course of auditory vocal hallucinations in childhood: 5-year follow-up study. Br J Psychiatry 2011;199:296-302.

47. Cannon TD, Cadenhead K, Cornblatt B et al. Prediction of psychosis in youth at high clinical risk: a multisite longitudinal study in North America. Arch Gen Psychiatry 2008;65:28-37.

48. Seidman LJ, Giuliano AJ, Meyer EC et al. Neuropsychology of the prodrome to psychosis in the NAPLS consortium: relationship to family history and conversion to psychosis. Arch Gen Psychiatry 2010;67:578-88.

49. Thompson A, Nelson B, Yung A. Predictive validity of clinical variables in the "at risk" for psychosis population: international comparison with results from the North American Prodrome Longitudinal Study. Schizophr Res 2011;126:51-7.

50. Yuen T, Chow EWC, Silversides CK et al. Premorbid adjustment and schizophrenia in individuals with 22q11.2 deletion syndrome. Schizophr Res 2013;151:221-5. 
Table 1 Descriptive characteristics of non-ultra high risk (UHR-) and ultra high risk (UHR+) participants at baseline

\begin{tabular}{|c|c|c|c|}
\hline & UHR- $(\mathrm{N}=67)$ & $\mathrm{UHR}+(\mathrm{N}=22)$ & Statistics \\
\hline Age (years), mean \pm SD (range) & $15.9 \pm 4.9(8-30)$ & $16.6 \pm 4.0(9-24)$ & $\mathrm{t}=-0.616, \mathrm{p}=0.539$ \\
\hline Gender, $\mathrm{N}$ females $(\%)$ & $38(56.7 \%)$ & $10(45.4 \%)$ & $\chi^{2}=0.845, p=0.358$ \\
\hline Any antipsychotics, N (\%) & $0(0 \%)$ & $5(22.7 \%)$ & $\chi^{2}=16.134, p<0.001$ \\
\hline SIPS P1, median (range) & $1.00(0-3)$ & $3.00(0-4)$ & $\mathrm{U}=1217.00, p<0.001$ \\
\hline SIPS P2, median (range) & $1.00(0-3)$ & $2.50(1-5)$ & $\mathrm{U}=1174.00, p<0.001$ \\
\hline SIPS P3, median (range) & $0.00(0-2)$ & $0.00(0-3)$ & $\mathrm{U}=857.50, \mathrm{p}=0.145$ \\
\hline SIPS P4, median (range) & $0.00(0-4)$ & $3.00(0-6)$ & $\mathrm{U}=1298.00, p<0.001$ \\
\hline SIPS P5, median (range) & $0.00(0-3)$ & $2.00(0-5)$ & $\mathrm{U}=1027.00, p=0.002$ \\
\hline Any Axis I diagnosis, N (\%) & $41(61.2 \%)$ & $16(72.7 \%)$ & $\chi^{2}=0.957, p=0.328$ \\
\hline Any anxiety disorder, N (\%) & $20(29.9 \%)$ & $13(59.1)$ & $\chi^{2}=6.069, p=0.014$ \\
\hline Any mood disorder, $\mathrm{N}(\%)$ & $15(22.4 \%)$ & $5(22.7 \%)$ & $\chi^{2}=0.001, p=0.974$ \\
\hline Any behavioral disorder, $\mathrm{N}(\%)$ & $17(25.4 \%)$ & $6(27.3 \%)$ & $\chi^{2}=0.031, p=0.860$ \\
\hline Any substance use, N (\%) & $1(1.5 \%)$ & $0(0 \%)$ & $\chi^{2}=0.332, p=0.564$ \\
\hline CGAS/GAF score, mean \pm SD & $63.0 \pm 11.3$ & $56.6 \pm 8.8$ & $U=484.50, p=0.016$ \\
\hline CGAS/GAF score $<70, \mathrm{~N}(\%)$ & $45(67.2 \%)$ & $19(86.4 \%)$ & $\chi^{2}=3.022, p=0.082$ \\
\hline Verbal IQ, mean \pm SD & $81.1 \pm 13.2$ & $77.3 \pm 10.8$ & $\mathrm{t}=1.227, \mathrm{p}=0.223$ \\
\hline Performance IQ, mean \pm SD & $77.1 \pm 11.9$ & $73.9 \pm 14.1$ & $\mathrm{t}=1.070, \mathrm{p}=0.288$ \\
\hline Full-scale IQ, mean \pm SD & $77.6 \pm 11.9$ & $74.1 \pm 11.9$ & $\mathrm{t}=1.208, \mathrm{p}=0.230$ \\
\hline $\begin{array}{l}\text { CBCL/ABCL internalizing t- } \\
\text { score, mean } \pm \text { SD }\end{array}$ & $62.7 \pm 11.0$ & $70.3 \pm 9.4$ & $\mathrm{U}=1036.5, p=0.004$ \\
\hline $\begin{array}{l}\text { CBCL/ABCL externalizing t- } \\
\text { score, mean } \pm \text { SD }\end{array}$ & $53.8 \pm 9.8$ & $60.9 \pm 10.8$ & $\mathrm{U}=968.5, \mathrm{p}=0.009$ \\
\hline
\end{tabular}




\begin{tabular}{l|lll}
\hline $\begin{array}{l}\text { CBCL/ABCL total problems t- } \\
\text { score, mean } \pm \text { SD }\end{array}$ & $62.1 \pm 10.7$ & $69.5 \pm 11.5$ & $\mathbf{U = 9 9 8 . 0 0 , p = 0 . 0 1 3}$ \\
\end{tabular}

SIPS - Structured Interview for Psychosis-Risk Syndromes, CGAS - Childhood Global Assessment Scale, GAF - Global Assessment of Functioning, CBCL - Child Behavior Checklist, ABCL Adult Behavior Checklist

Significant differences are highlighted in bold prints 
Table 2 Outcome at follow-up for non-ultra high risk (UHR-) and ultra high risk (UHR+) participants

UHR- participants $(\mathrm{N}=67)$

UHR-, N (\%)

$\mathrm{UHR}+, \mathrm{N}(\%)$

Psychotic disorder, N (\%)

$60(89.6 \%)$

$4(6 \%)$

$3(4.5 \%)$

UHR+ participants $(\mathrm{N}=22)$

UHR-, N (\%)

$\mathrm{UHR}+, \mathrm{N}(\%)$

Psychotic disorder, N (\%)

$10(45.5 \%)$

$6(27.3 \%)$

$6(27.3 \%)$ 
Table 3 Final Cox regression model

\begin{tabular}{l|cccccc}
\hline Predictors & $\boldsymbol{\beta}$ & SE & Wald (df) & $\mathbf{p}$ & $\mathbf{E x p}(\boldsymbol{\beta})$ & $\mathbf{9 5 \%} \mathbf{C I}$ \\
\hline $\begin{array}{l}\text { Any UHR criteria at } \\
\text { baseline }\end{array}$ & 1.544 & 0.748 & $4.266(1)$ & 0.039 & 4.685 & $1.082-20.286$ \\
\hline Baseline CGAS/GAF & -0.086 & 0.030 & $8.209(1)$ & 0.004 & 0.903 & $0.865-0.973$ \\
\hline
\end{tabular}

UHR - ultra high risk, CGAS - Childhood Global Assessment Scale, GAF - Global Assessment of Functioning 